\title{
Baixo peso ao nascer e condições maternas no pré-natal
}

\author{
LOW BIRTH WEIGHT AND MATERNAL CONDITIONS IN PRE-NATAL
}

BAJO PESO AL NACER Y CONDICIONES MATERNAS EN EL PERÍODO PRENATAL

\section{Áurea Tamami Minagawa', Rosângela Elaine Minéo Biagoline², Elizabeth Fujimori ${ }^{3}$, Ida Maria Vianna de Oliveira ${ }^{4}$, Ana Paula de Campos Araújo Moreira ${ }^{5}$, Luiza Dolores Saldaña Ortega ${ }^{6}$}

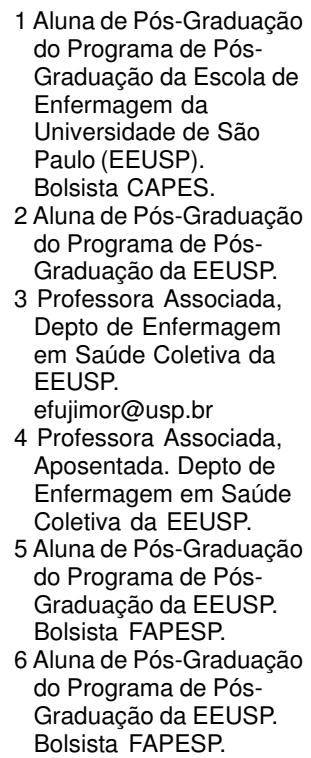
do Programa de PósGraduação da Escola de Enfermagem da Universidade de São Paulo (EEUSP). Bolsista CAPES

2 Aluna de Pós-Graduação do Programa de PósGraduação da EEUSP.

3 Professora Associada Depto de Enfermagem em Saúde Coletiva da EEUSP. efujimor@usp.br

4 Professora Associada, Aposentada. Depto de Enfermagem em Saúde Coletiva da EEUSP

5 Aluna de Pós-Graduação do Programa de PósGraduação da EEUSP. Bolsista FAPESP.

6 Aluna de Pós-Graduação do Programa de PósGraduação da EEUSP. Bolsista FAPESP.

\section{RESUMO}

Para verificar como o peso ao nascer se relaciona às condições maternas de trabalho, pré-natal, nutricionais (altura, peso inicial, final e ganho na gestação) e sócioeconômico-demográficas (idade, situação conjugal, escolaridade, renda familiar), o presente estudo foi realizado na área de abrangência do Hospital Universitário da Universidade de São Paulo e a amostra foi constituída por 101 crianças. A ocorrência de baixo peso ao nascer (BPN) foi de $5,1 \%$ e não se associou com o trabalho materno, nem com o pré-natal, porém mostrou associação significativa com as seguintes variáveis maternas: ganho de peso na gestação inferior a $7 \mathrm{~kg}$, idade menor que 20 anos e não ter companheiro. Apesar da baixa ocorrência de BPN, os resultados evidenciaram a importância do pré-natal para reduzir a ocorrência de BPN, especialmente por meio do controle do ganho de peso na gestação, controle da gestação na adolescência e também priorizando a atenção das mulheres sem companheiro.

\section{DESCRITORES}

Recém-nascido de baixo peso.

Trabalho feminino.

Condições sociais.

Cuidado pré-natal.

\section{ABSTRACT}

This study was carried in the area covered by the Hospital Universitário of the University of São Paulo in order to verify the relationship between birth weight and maternal work and prenatal, nutritional (height, initial and final weight and weight gain during gestation) and socioeconomicdemographic (age, marital status, level of education, family income) conditions. It was based on a sample of 101 children. The occurrence of low birth weight (LBW) $(5.1 \%)$ did not show association with maternal work nor with prenatal. On the other hand, LBW showed significant association with the following maternal variables: weight gain during the pregnancy under 7 kilograms, mother's age under 20 years old and marital status (single mothers). Despite the low occurrence of LBW, the results point out to the importance of taking measures to reduce LBW, in particular through the control of prenatal weight gain and prenatal among adolescents. Moreover, a policy of educating women, and in particular single women, about pregnancy, childbirth and nutrition seem an obvious necessity.

\section{KEY WORDS}

Infant, low birth weight.

Women, working.

Social conditions.

Prenatal care.

\section{RESUMEN}

El presente estudio realizado en la jurisdicción del Hospital Universitario de la Universidad de Sao Paulo tuvo como objetivo verificar cómo el peso al nacer se relaciona a las condiciones maternas de trabajo, el período prenatal, nutricionales (altura, peso inicial, final y ganancia en la gestación) y socio-económicodemográficas (edad, situación conyugal, escolaridad, ingreso familiar). La muestra estuvo constituida por 101 niños. La ocurrencia de bajo peso al nacer (BPN) fue del 5,1\% y no se asoció con el trabajo materno, ni con el prenatal, sin embargo mostró asociación significativa con las siguientes variables maternas: ganancia de peso en la gestación inferior a $7 \mathrm{~kg}$, edad menor a 20 años y no tener compañero. A pesar de la baja ocurrencia de BPN, los resultados evidenciaron la importancia del control prenatal para reducir la ocurrencia de BPN, especialmente por medio del control de ganancia de peso en la gestación, control de la gestación en la adolescencia y también priorizando la atención de las mujeres sin compañero.

\section{DESCRIPTORES}

Recien nacido de bajo peso.

Trabajo de mujeres.

Condiciones sociales.

Atención Prenatal. 


\section{INTRODUÇÃO}

O baixo peso ao nascer, conseqüência de um crescimento intra-uterino inadequado, de um menor período gestacional, ou da combinação de ambos, constitui o mais importante determinante da mortalidade neonatal, perinatal e infantil ${ }^{(1-3)}$

Vários estudos mostram que o baixo peso ao nascer está inequivocamente associado ao risco de adoecer e morrer no primeiro ano de vida ${ }^{(2,4)}$. Além disso, estudos epidemiológicos retrospectivos evidenciaram, na década passada, associação entre subnutrição intra-uterina e doenças crônicas na vida adulta, tais como doença cardiovascular, hipertensão arterial, diabetes melitos não insulinodependente e câncer ${ }^{(8-11)}$.

Assim, no campo da saúde coletiva, a preocupação com a ocorrência do baixo peso ao nascer se apóia tanto no que ele representa para a morbidade e mortalidade infantil, quanto na freqüência com que o mesmo ocorre. Segundo o Fundo das Nações Unidas para a Infância (Unicef) ${ }^{(12)}$, em nível mundial, $14 \%$ das crianças nasceram com baixo peso no período de 1995-2000. Essa taxa variou de acordo com as condições de desenvolvimento social de cada localidade, sendo que a mais alta foi encontrada em Bangladesh $(30 \%)^{(12)}$. No Brasil, dados da Pesquisa Nacional sobre Saúde e Nutrição indicaram que a incidência de baixo peso ao nascer era de $11,0 \%$, variando de $5,9 \%$ a $12,5 \%$ conforme a renda per capita $^{(13)}$.

A Organização Mundial da Saúde estima que nos países desenvolvidos, cerca de dois terços dos recém nascidos de baixo peso sejam prematuros, enquanto um terço é pequeno para idade gestacional. Essa relação se inverte nos países pobres onde aproximadamente $75 \%$ dos recém-nascidos de baixo peso são pequenos para a idade gestacional ${ }^{(14)}$. Nesses países, o baixo peso ao nascer quase sempre está fortemente associado às condições de pobreza, má nutrição crônica materna e consumo alimentar insuficiente ${ }^{(15)}$.

Tendo como perspectiva a determinação social do processo saúde-doença ${ }^{(16)}$, pode-se afirmar que o complexo encadeamento da rede de variáveis envolvidas na ocorrência do baixo peso ao nascer tem sua gênese nas precárias condições de vida e trabalho em que nascem, crescem e vivem parcelas consideráveis de nossa população. Dessa maneira, os numerosos determinantes do baixo peso ao nascer, podem ser considerados quase totalmente mediados pelas condições de $\operatorname{vida}^{(5,17)}$.

No Brasil, a precária situação perinatal guarda relação com o acesso à assistência pré-natal e ao parto, e evidencia as dificuldades impostas socialmente à gestante, impossibilitando-a de freqüentar o pré-natal dentro de condições adequadas. Entre as dificuldades referidas, encontra-se o horá- rio de funcionamento dos serviços que, muitas vezes, coincide com o de trabalho das gestantes ${ }^{(18)}$.

Considerando a inserção da mulher brasileira no mercado de trabalho, constata-se que a participação feminina na população economicamente ativa aumentou sensivelmente, passando de 32,9\% em 1980 para 41,0\% em 2001 ${ }^{(19-20)}$. Entre 1970 e 1990, a mão-de-obra feminina cresceu $180 \%$, enquanto a masculina cresceu $71 \%{ }^{(21)}$. Vale salientar que estes dados sofrem um sub-registro considerável, especialmente nos trabalhos informais e nas zonas rurais, como também acontece em outros países da América Latina ${ }^{(21-22)}$.

A ampliação dos papéis femininos na esfera pública tem se refletido no âmbito privado da família, espaço tradicional de atuação e produção das mulheres ${ }^{(23)}$. Neste espaço ainda é ratificada a importância da mulher, no que se refere à reprodução do núcleo familiar, seu bem estar geral ou mesmo o desenvolvimento infantil (24).

O pressuposto de que a inserção materna no trabalho remunerado, enquanto formas de trabalhar da família, ao influenciar as formas de viver, determinaria pré-condições para o fortalecimento/desgaste, evidenciadas no seu processo gestacional e no peso ao nascer de crianças, mediados pela inserção no pré-natal, impulsionou o desenvolvimento deste estudo. Assim, foi objetivo deste trabalho verificar como o peso ao nascer das crianças se relaciona com a inserção materna no trabalho remunerado e no pré-natal, e com as condições nutricionais e sócio-econômicas das mães.

\section{METODOLOGIA}

Este estudo, de natureza transversal-descritivo, integrou uma ampla investigação denominada "Morbidade referida nas famílias de um Sistema Local de Saúde - Distrito de Saúde do Butantã e UBS Vila Nova Jaguaré - e a relação com suas formas de trabalhar e de viver"(a) (Projeto-Morbidade). Utilizando-se a técnica de Amostragem Casual Estratificada Proporcional, foram sorteados um total de 349 domicílios (para um erro de aproximadamente $3 \%$ e $10 \%$ de perdas) distribuídos proporcionalmente ao número de habitantes das áreas de abrangência das 14 Unidades de saúde que conformavam a área de abrangência do Hospital Universitário da Universidade de São Paulo. O sorteio foi feito com a utilização de mapa da região dividido em $\mathrm{cm}^{2}$, com sorteio de abscissas e ordenadas que delimitavam a rua, que então tinha um número da Tabela de Números Aleatórios Simples sorteado para definir o número do domicílio a ser visitado.

\footnotetext{
(a) Queiroz VM, Salum MJ, Greco RM, Gryschek ALFPL. Contribuição para a intervenção em perfis epidemiológicos de grupos homogêneos que ocupam a área de abrangência do Hospital Universitário: morbidade referida nas famílias do Distrito de Saúde do Butantã e da UBS Vila Nova Jaguaré e a relação com suas formas de trabalhar e de viver. São Paulo: EEUSP/Depto de Enfermagem em Saúde Coletiva; 1996.
} 
A amostra do presente estudo refere-se a 101 crianças pertencentes a 80 famílias em que havia menores de 7 anos. Todos os domicílios foram visitados por uma equipe de entrevistadores treinados, que utilizaram um instrumento com questões abertas e fechadas.

Como os dados foram coletados no ano de 1996, o projeto não foi submetido a Comitê de Ética. No entanto, todos os aspectos éticos foram contemplados, ou seja, antes da coleta de dados, a mãe da criança foi informada sobre a justificativa, os objetivos e os procedimentos utilizados no estudo, assegurando-lhe o direito de participar ou não da pesquisa, e os dados só foram colhidos mediante o consentimento da entrevistada.

O estado nutricional materno foi analisado, considerando o peso pré-gestacional, altura, peso final e ganho de peso durante a gravidez, todos referidos. A avaliação foi feita a partir do índice de massa corporal (IMC) ${ }^{(25)}$, calculado por meio da relação peso $(\mathrm{kg}) /$ altura $^{(2)}(\mathrm{m})$, utilizando-se quatro categorias para classificação: baixo peso (IMC<19,8), peso normal $(19,8 \geq$ IMC $<26,0)$, sobrepeso $(26 \geq$ IMC $<29)$ e obesidade (IMC $\geq 29$ ) ${ }^{(26)}$. Utilizou-se $7 \mathrm{~kg}$ como ponto de corte para o ganho mínimo de peso durante a gestação ${ }^{(27)}$, tendo em vista o baixo percentual de mulheres obesas (2\%). Foram consideradas com Baixo Peso ao Nascer (BPN), as crianças nascidas com peso inferior a $2500 \mathrm{~g}^{(28)}$.

$\mathrm{Na}$ análise estatística, utilizou-se o programa EPI-INFO e os testes ANOVA e $\mathrm{X}^{2}$ (Fisher, quando $\mathrm{n}<5$ ), adotando-se um nível de significância de $5 \%$.

\section{RESULTADOS E DISCUSSÃO}

Estudo $^{(29)}$ destaca a necessidade não só de cuidar da criança com BPN, mas especialmente de prevenir esta vulnerabilidade. Assim, o conhecimento da prevalência de BPN, assim como seus determinantes, possibilita a priorização de intervenções de enfermagem no coletivo, principalmente nos grupos vulneráveis.

Do total das 101 crianças analisadas, duas não tiveram o seu peso informado. Observou-se uma ocorrência de BPN igual a 5,1\%. Essa proporção assemelha-se à de países desenvolvidos, pois em nossa região, a taxa gira em torno de 9\%: no Município de São Paulo, em 2000, $8,9 \%$ dos nascidos vivos tiveram $\mathrm{BPN}$ e na região $\mathrm{Su}-$ deste do Brasil, 8,5\%(30). Em nível mundial, dados do Unicef $^{(12)}$ revelam uma tendência de queda na porcentagem de crianças com BPN, porém com diferenças substanciais, de acordo com o nível de riqueza: 7,0\% nos países industrializados, 14,0\% nos países em desenvolvimento e $18,0 \%$ naqueles menos desenvolvidos, no período de 1995-2000.

A Tabela 1 apresenta a distribuição do peso ao nascer em relação à inserção materna no trabalho remunerado e no pré-natal. Observa-se que a maior parte das mães estavam inseridas no mercado de trabalho durante a gestação $(55,6 \%)$ e realizaram o pré-natal $(90,9 \%)$. Embora a proporção de BPN tenha sido mínima entre as mulheres trabalhadoras $(1,8 \%)$, em relação às não trabalhadoras $(9,1 \%)$, não se encontrou associação significativa entre o trabalho materno e o peso ao nascer $(\mathrm{P}>0,05)$. Em relação ao pré-natal, das cinco mães de crianças que apresentaram BPN apenas uma não recebeu atendimento pré-natal, justificando os resultados estatisticamente não significativos, pois a falta ou deficiência da atenção pré-natal tem sido evidenciada como fator explicativo e determinante do baixo peso ao nascer ${ }^{(13,31-32)}$. O risco relativo para BPN era de 2,6 para mães com três consultas ou menos de pré-natal, em relação às mães com sete ou mais consultas ${ }^{(3)}$.

Tabela 1 - Distribuição do peso ao nascer, segundo a inserção materna no trabalho remunerado e no pré-natal* - São Paulo - 1996-1997

\begin{tabular}{|c|c|c|c|}
\hline \multirow[b]{2}{*}{ Inserção materna } & \multicolumn{3}{|c|}{ Peso ao nascer } \\
\hline & $\begin{array}{c}\text { Baixo } \\
\text { n(\%) }\end{array}$ & $\begin{array}{c}\text { Normal } \\
\mathrm{n}(\%)\end{array}$ & $\begin{array}{c}\text { Total } \\
\text { n(\%) }\end{array}$ \\
\hline Trabalho ${ }^{\text {NS }}$ & $5(5,1)$ & $94(94,9)$ & $99(100,0)$ \\
\hline Inseridas & $1(1,8)$ & $54(98,2)$ & $55(55,6)$ \\
\hline Não inseridas & $4(9,1)$ & $44(90,9)$ & $44(44,4)$ \\
\hline Pré-Natal ${ }^{\mathrm{NS}}$ & $5(5,1)$ & $94(100,0)$ & $99(100,0)$ \\
\hline Inseridas & $4(4,4)$ & $86(95,6)$ & $90(90,9)$ \\
\hline Não inseridas & $1(11,1)$ & $8(88,9)$ & $9(9,1)$ \\
\hline
\end{tabular}

* Inserida no trabalho durante a gestação; não se obteve informações para todas as crianças;

Ns: Diferença estatisticamente não significativa $(P>0,05)$.

As Tabelas 2 e 3 mostram a distribuição do BPN em relação ao estado nutricional materno. Constatou-se que o estado nutricional materno representado pela altura, peso e IMC pré-gestacional, peso final e ganho de peso na gestação, não se relacionou com o BPN, conforme o observado em outras investigações ${ }^{(13,31-33)}$. Pelo contrário, verificou-se que as mães de crianças com BPN apresentaram altura média e peso inicial maior, o mesmo ocorrendo com o IMC prégestacional que apresentou-se ligeiramente maior, porém sem diferença significativa. 
Embora outro pesquisador ${ }^{(33)}$ também não tenha encontrado relação estatisticamente significativa entre a adequação peso-altura materna e BPN, o autor encontrou maior freqüência de BPN entre mães com peso pré-gestacional menor que $45 \mathrm{~kg}$, em relação àquelas com $70 \mathrm{~kg}$ ou mais.

Tabela 2 - Ocorrência de BPN segundo dados antropométricos maternos: distribuição percentual*- São Paulo - 1996-1997

\begin{tabular}{|c|c|c|c|}
\hline \multirow{2}{*}{ Variáveis } & \multicolumn{3}{|c|}{ Peso ao nascer } \\
\hline & $\begin{array}{c}\text { Baixo } \\
\text { n (\%) }\end{array}$ & $\begin{array}{c}\text { Normal } \\
\text { n (\%) }\end{array}$ & $\begin{array}{l}\text { Total } \\
\text { n (\%) }\end{array}$ \\
\hline Altura $(\mathrm{cm})^{\mathrm{NS}}$ & $5(5,1)$ & $94(94,9)$ & $99(100,0)$ \\
\hline$<150$ & - & $11(100,0)$ & $11(100,0)$ \\
\hline $150-159$ & $2(6,9)$ & $27(93,1)$ & $29(100,0)$ \\
\hline$>=160$ & $3(5,1)$ & $56(94,9)$ & $59(100,0)$ \\
\hline IMC pré-gestacional ${ }^{\mathrm{NS}}$ & $5(5,3)$ & $89(94,7)$ & $94(100,0)$ \\
\hline Baixo peso & - & $20(100,0)$ & $20(100,0)$ \\
\hline Peso normal & $4(6,5)$ & $58(93,5)$ & $62(100,0)$ \\
\hline Sobrepeso e Obesidade & $1(8,3)$ & $11(91,7)$ & $12(100,0)$ \\
\hline Peso inicial $(\mathrm{Kg})^{\mathrm{NS}}$ & $5(5,2)$ & $91(94,8)$ & $96(100,0)$ \\
\hline$<49$ & - & $17(100,0)$ & $17(100,0)$ \\
\hline $49-60,9$ & $4(7,7)$ & $48(92,3)$ & $52(100,0)$ \\
\hline$>=61$ & $1(3,7)$ & $26(96,3)$ & $27(100,0)$ \\
\hline Peso final $(\mathrm{Kg})^{\mathrm{NS}}$ & $5(5,4)$ & $88(94,6)$ & $93(100,0)$ \\
\hline$<55$ & - & $6(100,0)$ & $6(100,0)$ \\
\hline $55-74,9$ & $5(8,1)$ & $57(91,9)$ & $62(100,0)$ \\
\hline$>=75$ & - & $25(100,0)$ & $25(100,0)$ \\
\hline Ganho de peso $(\mathrm{Kg})^{* *}$ & $5(5,1)$ & $94(94,9)$ & $99(100,0)$ \\
\hline$<7$ & $3(15,8)$ & $16(84,2)$ & $19(100,0)$ \\
\hline $7-12,9$ & $2(2,5)$ & $78(97,5)$ & $80(100,0)$ \\
\hline
\end{tabular}

* Não se obteve informações para todas as mães;

Ns: diferença estatisticamente não significativa $(P>0,05)$;

** diferença estatisticamente significativa: $\mathrm{P}<0,05$ (teste $\mathrm{X}^{2}$ e para $\mathrm{n}<5$ teste Fisher).

Tabela 3 - Ocorrência de BPN segundo dados antropométricos maternos: médias*- São Paulo - 1996-1997

\begin{tabular}{|c|c|c|c|}
\hline \multirow{2}{*}{$\begin{array}{l}\text { Variáveis } \\
\text { antropométricas }\end{array}$} & \multicolumn{3}{|c|}{ Peso ao nascer } \\
\hline & $\begin{array}{c}\text { Baixo } \\
\text { Média + DP }\end{array}$ & $\begin{array}{c}\text { Normal } \\
\text { Média + DP }\end{array}$ & $\begin{array}{c}\text { Total } \\
\text { Média }+\mathrm{DP}\end{array}$ \\
\hline Altura $(\mathrm{cm})^{\mathrm{NS}}$ & $161,8+5,9$ & $159,4+6,5$ & $159,4+6,5$ \\
\hline IMC pré-gestacional ${ }^{\mathrm{NS}}$ & $22,7+2,6$ & $22,1+3,2$ & $22,1+3,1$ \\
\hline \multicolumn{4}{|l|}{ Peso na gestação (Kg) } \\
\hline Inicial NS & $59,6+7,4$ & $54,4+8,6$ & $56,4+8,5$ \\
\hline Final Ns & $67,0+4,5$ & $69,3+10,8$ & $68,9+10,6$ \\
\hline Ganho ** & $7,4+5,4$ & $12,9+6,7$ & $12,4+6,8$ \\
\hline
\end{tabular}

*Não se obteve informações para todas as mães;

Ns: diferença estatisticamente não significativa $(P>0,05)$;

** diferença estatisticamente significativa: $\mathrm{P}<0,05$ (teste ANOVA).

Da mesma forma, pesquisadores ${ }^{(32)}$, estudando os fatores de risco para BPN em casuística de 20850 nascidos vivos do Município de São Paulo, observaram que o peso materno inferior a $50 \mathrm{~kg}$ elevou significativamente (1,9 vezes) a incidência de BPN. E outro estudo desenvolvido no Município de Pelotas reiterou-se esses achados, demonstrando que o peso materno era um dos melhores fatores prognósticos do BPN no início da gestação ${ }^{(31)}$.

Com relação à altura, constataram que mulheres com estatura inferior a $150 \mathrm{~cm}$ tinham maior probabilidade de dar à luz recémnascidos com baixo peso $^{(34)}$, resultado também constatado por pesquisadores ${ }^{(35)}$ na Jamaica, que encontraram aumento progressivo do peso ao nascer com o aumento da altura materna.

No presente estudo, apenas o ganho de peso durante a gestação associou-se com o peso ao nascer: mães de crianças com BPN apresentaram ganho de peso significativamente menor que mães de crianças com peso ao nascer normal $(P<0,05)$ (Tabelas 2 e 3$)$.

Embora não se tenha verificado uma associação significativa entre o peso ao nascer e a inserção no pré-natal (Ta- 
bela 1), este estudo encontrou uma relação significativa $(P<0,05)$ entre o ganho de peso materno e a inserção no prénatal (Figura 1). Como pode ser observado, das mulheres que não fizeram o pré-natal, $60 \%$ ganharam menos que $7 \mathrm{~kg}$, enquanto das que fizeram pré-natal, apenas 16,4\% apresentaram ganho de peso insuficiente.

O BPN relaciona-se com a desnutrição materna e o ganho de peso insuficiente durante a gestação em populações sócio-econômicamente desfavorecidas ${ }^{(36)}$. Para esses autores, o risco de nascimento de crianças com baixo peso pode ser diminuído consideravelmente por meio da intervenção nutricional em gestantes mal nutridas, sendo que a avaliação do ganho ponderal durante a gestação constitui uma das medidas mais importantes a ser efetuada pelos profissionais de saúde durante o atendimento pré-natal. A vigilância estrita do ganho de peso e a suplementação alimentar nos casos de maior necessidade devem integrar medidas preventivas do $\mathrm{BPN}^{(31)}$. A deficiência no atendimento prénatal umas das causas da maior incidência de $\mathrm{BPN}^{(13)}$.

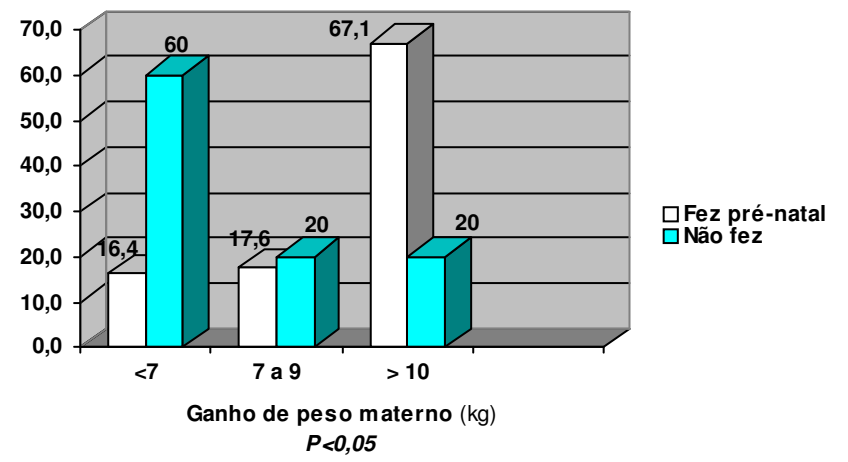

Figura 1 - Distribuição das mães segundo sua inserção no pré-natal e ganho de peso na gestação - São Paulo
Ao se analisar a ocorrência do BPN segundo características sócio-econômicas maternas (Tabela 4), observouse uma diferença estatisticamente significativa no que se refere à situação conjugal da gestante e o peso ao nascer das crianças. No grupo de mães sem companheiro no momento do parto, a proporção de crianças com BPN foi significativamente maior $(21,4 \%)$ que no grupo de mães com companheiro $(2,4 \%)(P<0,05)$. Talvez esse fato possa ser justificado pela idade materna no parto, uma vez que as mães de crianças com BPN eram significativamente mais jovens (quatro em cinco, ou seja, 80,0\% eram adolescentes, com menos de 20 anos). A incidência de $25 \%$ de BPN entre mães com menos de 20 anos foi de 1,5 a 2,5 vezes maior que as relatadas por outros estudos que apresentaram resultados de $(10,3 \%)^{(37)},(12,4 \%)^{(38)}$ e $(15,3 \%)^{(33)}$ respectivamente.

$\mathrm{Na}$ adolescência, à necessidade nutricional aumentada, somam-se os problemas de natureza fisiológica e sócio-culturais $^{(39)}$. Citam-se estudos que associam o BPN e a adolescência materna a fatores psico-sociais e biológicos, entre os quais se incluem a situação sócio-econômica e o acesso tanto às informações quanto aos serviços de pré-natal ${ }^{(37)}$. A gravidez precoce nos grupos sociais mais carentes pode resultar em início tardio ou até mesmo ausência de atendimento pré-natal, pressupondo maior probabilidade de complicações obstétricas, se forem consideradas as precárias condições sanitárias em que ocorrem a gestação e o parto ${ }^{(40)}$.

Tabela 4 - Incidência de BPN, segundo características sócio-econômicas maternas*- São Paulo - 1996-1997

\begin{tabular}{|c|c|c|c|}
\hline \multirow[b]{2}{*}{ Variáveis } & \multicolumn{3}{|c|}{ Peso ao nascer } \\
\hline & $\begin{array}{c}\text { Baixo } \\
\text { n (\%) }\end{array}$ & $\begin{array}{c}\text { Normal } \\
\mathrm{n}(\%)\end{array}$ & $\begin{array}{l}\text { Total } \\
\text { n (\%) }\end{array}$ \\
\hline Situação conjugal** & $5(5,1)$ & $94(94,9)$ & $99(100,0)$ \\
\hline Com companheiro & $2(2,4)$ & $83(97,6)$ & $85(100,0)$ \\
\hline Sem companheiro & $3(21,4)$ & $11(78,6)$ & $14(100,0)$ \\
\hline Idade no parto (anos) ${ }^{* *}$ & $5(5,1)$ & $94(94,9)$ & $99(100,0)$ \\
\hline$<20$ & $4(25,0)$ & $12(75,0)$ & $16(100,0)$ \\
\hline $20-24$ & $1(3,4)$ & $28(96,6)$ & $29(100,0)$ \\
\hline$>=25$ & - & $54(100,0)$ & $54(100,0)$ \\
\hline Escolaridade (anos de estudo) ${ }^{\mathrm{NS}}$ & $5(5,1)$ & $94(94,9)$ & $99(100,0)$ \\
\hline$<=8$ & $4(7,3)$ & $51(92,7)$ & $55(100,0)$ \\
\hline$>8$ & $1(2,3)$ & $43(97,7)$ & $44(100,0)$ \\
\hline Renda familiar (SM) ${ }^{\mathrm{NS}}$ & $4(4,2)$ & $91(95,8)$ & $95(100,0)$ \\
\hline$<=1$ & $1(5,9)$ & $16(94,1)$ & $17(100,0)$ \\
\hline $1,1-3$ & - & $5(100,0)$ & $5(100,0)$ \\
\hline $3,1-6$ & - & $14(100,0)$ & $14(100,0)$ \\
\hline $6,1-9,9$ & $2(12,5)$ & $14(87,5)$ & $16(100,0)$ \\
\hline$>=10$ & $1(2,3)$ & $42(97,7)$ & $43(100,0)$ \\
\hline
\end{tabular}

* Não se obteve informações para todas as mães;

${ }^{* *}$ Diferença estatisticamente significativa: $\mathrm{P}<0,05$ (teste $\mathrm{X}^{2}$ e para $\mathrm{n}<5$ teste Fisher);

Ns: diferença estatisticamente não significativa $(P>0,05)$;

Rev Esc Enferm USP
$2006 ; 40(4): 548-54$.
www.ee.usp.br/reeusp/


Em relação à escolaridade, a Tabela 4 também aponta diferença na proporção de BPN (7,3\% entre mães com 8 anos ou menos de estudo, comparado a $2,3 \%$ entre mães com mais de 8 anos de estudo), porém sem significância estatística. Encontrou-se duas vezes mais filhos com BPN entre mães sem nenhuma instrução, em relação àquelas com 9 anos ou mais de estudo $(13,5 \% \text { e } 6,2 \% \text {, respectivamente })^{(38)}$.

Considerando que a renda familiar se constitui na somatória dos rendimentos resultantes do trabalho, entre os quais se inclui os do trabalho materno, da mesma forma que não se constatou associação significativa entre peso ao nascer e inserção materna no mercado de trabalho, conforme já referido, também não se verificou diferença significativa entre a renda familiar de crianças com BPN e peso normal.

A Figura 2 mostra a análise entre a inserção materna no trabalho remunerado e o ganho de peso durante a gestação. Observa-se que $15,8 \%$ das mulheres que trabalhavam ganharam menos que $7 \mathrm{~kg}$, enquanto essa proporção foi de $25,0 \%$ entre as que não trabalhavam, porém a diferença não foi significativa.

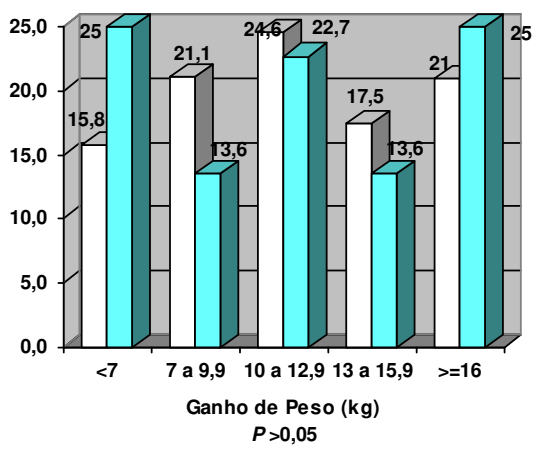

$\square$ Mães inseridas $\square$ Mães não inseridas

Figura 2 - Distribuição das mães segundo sua inserção no trabalho remunerado e seu ganho de peso na gestação - São Paulo

\section{CONCLUSÕES}

Embora não se tenha comprovado a hipótese de que a inserção materna no trabalho remunerado, enquanto formas de trabalhar da família, ao influenciar as formas de viver, determinaria as pré-condições para o desgaste - ganho de peso inadequado durante o processo gestacional e baixo peso ao nascer -, pode-se concluir que:

- peso ao nascer mostrou associação significativa com o ganho de peso materno durante a gestação: as mães de crianças com BPN ganharam significativamente menos peso durante a gestação;

- o ganho de peso na gestação mostrou associação significativa com a inserção no pré-natal: maior proporção de mães que não fizeram o pré-natal ganharam peso insuficiente;

- o peso ao nascer mostrou associação significativa com algumas variáveis sócio-econômico-demográficas maternas: as mães de crianças com BPN eram adolescentes e não tinham companheiro no momento do parto.

Assim, os resultados obtidos evidenciam a importância do pré-natal, que mesmo não apresentando associação direta com o BPN neste estudo, é imprescindível para controlar o ganho de peso durante a gestação. Além disso, os resultados evidenciam também a importância de se priorizar a atenção prestada a gestantes adolescentes e mulheres sem companheiro.

(5) Escamilla RP, Pollitt E. Causas y consecuencias del retraso del crescimiento intrauterino en América Latina. Bol Of Sanit Panam. 1992;112(6):473-93.

(6) Lira PIC, Ashworth A, Morris SS. Low birth weight and morbidity from diarrhea and respiratory infextion in northeast Brazil. J Pediatr. 1996;128(4):497-504.

(7) Almeida MF, Jorge MHPM. Pequenos para idade gestacional: fator de risco para mortalidade neonatal. Rev Saúde Pública. 1998;32(3):217-24.

(8) Barker DJ, Gluckman PD, Godfrey KM, Harding JE, Owens JA, Robinson JS. Fetal nutrition and cardiovascular disease in adult life. Lancet. 1993;341(8850):938-41. 
(9) Lopes AAS, Port FK. The low birth weight hypothesis as a plausible explanation for the black white differences in hypertension, non-insulin-dependent diabetes and end-stage renal disease. Am J Kidney Dis. 1995;25(2):350-6.

(10) Michels KB, Trichopoulos D, Robins JM, Rosner BA, Manson J, Hunter DJ, et al. Birth weight as a risk factor for breast cancer. Lancet. 1996;348(9041):1542-6.

(11) Leon DA. Fetal growth and adult disease. Eur J Clin Nutr. 1998;52(suppl. 1):S72-82.

(12) Fundo das Nações Unidas para a Infância (UNICEF). Situação mundial da infância 2003. Brasília: Unicef; 2003.

(13) Monteiro MFG. Baixo peso ao nascer. In: Monteiro MFG, Cervini R, editores. Perfil estatístico de crianças e mães no Brasil: aspectos de saúde e nutrição de crianças e mães no Brasil, 1989. Rio de Janeiro: IBGE; 1992. p. 43-60.

(14) Falkner F, Holzgreve W, Schloo RH. Prenatal influences on posnatal growth: overview and pointers for needed research. Eur J Clin Nutr. 1994;48(suppl. 1):S22-24.

(15) Prada JA, Tsang RC. Biological mechanisms of environmentally induced causes of IUGR. Eur J Clin Nutr. 1998;52(suppl. 1): S27-8.

(16) Laurell AC. La salud-enfermedad como proceso social. Cuad Méd Soc. 1982;19:7-19.

(17) Bernstein PS, Divon MY. Etiologies of fetal growth restriction. Clin Obstetr Gynecol. 1997;40(4):723-9.

(18) Bonadio IC. Ser tratada como gente: a vivência de mulheres atendidas no serviço de pré-natal de uma instituição filantrópica [tese]. São Paulo: Escola de Enfermagem da USP; 1996.

(19) Aquino EML GMS, Marinho LFB. Mulher, saúde e trabalho no Brasil: desafios para um novo agir. Cad Saúde Pública. 1995;11(2):281-90.

(20) Departamento Intersindical de Estatística e Estudos Socioeconômicos (DIEESE). Pesquisas temáticas. Mulheres trabalhadoras: discriminação e desigualdade no mercado de trabalho[online]. São Paulo; 2001. Disponível em: <http:// www.dieese. org.br/> [Acesso em 7 jan. 2004].

(21) Valdes T, Gomariz E, editores. Mulheres latino-americanas em dados. Santiago do Chile: Instituto de la Mujer; 1993.

(22) Bruschini C. Mulher e trabalho: uma avaliação da década da mulher. São Paulo: Nobel; 1985.

(23) Instituto Brasileiro de Geografia e Estatística (IBGE). Departamento de Estatística e Indicadores Sociais. Crianças e adolescentes, v.4 , Rio de Janeiro; 1989.

(24) Facchini LA. Trabalho materno e ganho de peso infantil. Pelotas: Universitária; 1995.

(25) Anjos LA. Índice de massa corporal (massa corporal.estatura² $\left.{ }^{2}\right)$ como indicador do estado nutricional de adultos: revisão da literatura. Rev Saúde Pública. 1992;26(6):431-6.

(26) Institute of Medicine. National Academy of Sciences. Food and Nutricional Board. Nutritional during pregnancy. Part I: weight gain. Part II: Nutrition supplements. Washington, DC: National Academy Press; 1990.
(27) Worthington-Roberts BS, Vermeersch J, Williams SR. Nutrição na gravidez e na lactação. Rio de Janeiro: Interamericana; 1986.

(28) Organização Mundial da Saúde (OMS). CID-10: classificação internacional de doenças e problemas relacionados à saúde. $10^{a}$ rev. São Paulo: Ed Universidade de São Paulo; 1998.

(29) Mello DF, Rocha SMM, Martins DC, Chiozi SZ. Cuidados maternos a crianças de baixo peso ao nascer. Rev Esc Enferm USP. 2002;36(3):262-9.

(30) Brasil. Ministério da Saúde. Datasus. Informações de saúde; 2001. Estatísticas vitais - mortalidade e nascidos vivos. [online]. Brasília, 2004. Disponível na internet: www.datasus. gov.br [Acesso em 13 fev. 2004].

(31) Barros FC, Victora CG, Vaughan JP, Estanislau HJ. Bajo peso al nacer en el municipio de Pelotas, Brasil: factores de riesgo. Bol Of Sanit Panam. 1987;102(6):541-53.

(32) Benicio MHd'A, Monteiro CA, Pacheco JMS, Castilho EA, Lamonica IMR. Análise multivariada de fatores de risco para o baixo peso ao nascer em nascidos vivos do município de São Paulo, SP (Brasil). Rev. Saúde Pública. 1985;19(4):311-20.

(33) Rocha JA. Baixo peso, peso insuficiente e peso adequado ao nascer, em 5940 nascidos vivos na cidade de Recife: associação com algumas variáveis maternas. J Pediatr. 1991; 67(9/10): 297-304.

(34) Siqueira AAF, Ciari JRC, Tanaka AC d'A, Montelone PPR, Arruda JJG. Influência da altura e ganho de peso maternos e da idade gestacional sobre o peso do recém-nascido: estudo de 3 grupos de gestantes normais. Rev Saúde Pública. 1975(9):331-42.

(35) Thame M, Wilky RJ, McFarlane-Anderson N, Bennett FI, Forrester TE. Relationship between maternal nutritional status and infant's weight and body proportions at birth. Eur J Clin Nutr. 1997; 51(3):134-8.

(36) Lima CMG, Pelá NTR. Estudo com gestantes atendidas em um Centro Municipal de Saúde da periferia de Botucatu - São Paulo: relação entre baixo peso materno e baixo peso ao nascer do concepto. Rev Lat Am Enferm. 1995;3(1):79-94.

(37) Costa CE, Gotlieb SLD. Estudo epidemiológico do peso ao nascer a partir da Declaração de Nascido Vivo. Rev Saúde Pública. 1998; 32(4): 328-34.

(38) Victora CG, Barros FC, Vaughan JP. Epidemiologia da desigualdade: um estudo longitudinal de 6000 crianças brasileiras. São Paulo: Hucitec; 1989.

(39) Silva AAM, Barbieri MA, Bettiol H, Bó CRD, Mucillo G, Gomes UA. Saúde perinatal: baixo peso e classe social. Rev Saúde Pública. 1991;25(2):87-95.

(40) Oliveira MAC, Egry EY. A adolescência enquanto fenômeno social: possibilidades e necessidades de investigação científica em enfermagem. Rev Bras Enferm. 1993;46(1):63-7. 